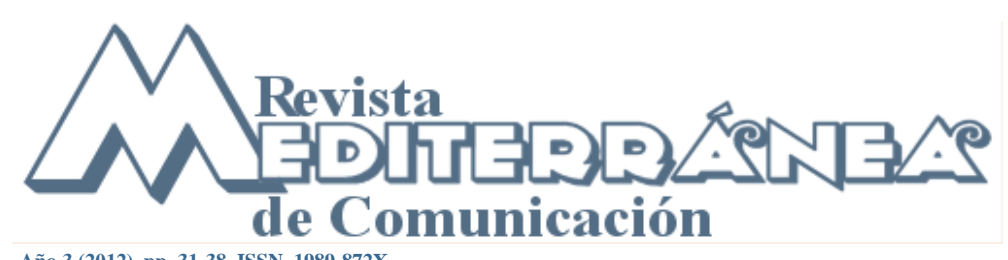

Año 3 (2012), pp. 31-38 ISSN 1989-872X

Fecha de recepción: 03/12/2011; Fecha de publicación: 05/02/2012

\title{
Carl I. Hovland en las revistas científicas de comunicación en España (2000-2010).
}

\section{Carl I. Hovland in communication journals in Spain (2000- 2010).}

\author{
José A. Ruiz San Román \\ Universidad Complutense Madrid - España \\ jar.sanroman@gmail.com \\ Mariam González \\ CES Villanueva, Madrid- España
}

\begin{abstract}
Resumen
El artículo estudia la repercusión de la obra de Carl I.Hovland (1912-1961) en la investigación científica en comunicación en España en los últimos diez años. Se ha realizado un análisis de contenido cuantitativo de revistas científicas sobre comunicación publicadas en España (Anàlisi: quaderns de comunicació i cultura, Comunicación y Sociedad, Comunicar. Revista de Medios de Comunicación y Educación, Estudios sobre el Mensaje Periodístico, Revista Latina de Comunicación Social, Telos: Cuadernos de comunicación e innovación y Zer. Revista de Estudios de Comunicación). Se trata de las denominadas revistas fuente por In RECS. Se ha trabajado sobre una muestra de 1408 artículos en revistas científicas. En las conclusiones se subraya la escasa presencia de Hovland y de su propuesta metodológica de investigar mediante experimentos.
\end{abstract}

\section{Abstract}

This article studies the influence of Carl I.Hovland (1912-1961) in communication research in Spain during last ten years. We use quantitative content analyse of spanish journals of communication (Anàlisi: quaderns de comunicació i cultura, Comunicación y Sociedad, Comunicar. Revista de Medios de Comunicación y Educación, Estudios sobre el Mensaje Periodístico, Revista Latina de Comunicación Social, Telos: Cuadernos de comunicación e innovación y Zer. Revista de Estudios de Comunicación. All of them are journals considered by In Recs as source. We use sample of 1408 articles. We underline the scanty presence of Hovland and of his methodological offer of investigating by means of experiments.

\section{Palabras claves}

Hovland; experimentos; investigación; comunicación; revistas científicas; España. 


\section{Key Words}

Hovland; experiments; research; communication; scientific journals; Spain.

Sumario: 1. Introducción. Hovland en la investigación en comunicación. 2. Experimentos aplicados a la comunicación. 3. Objeto y metodología. 4. Resultados. 5. Discusión y conclusión. 6. Bibliografía.

Summary: 1. Introduction. Hovland in communication research. 2. Experiments applied to communication. 3. Purpose and methodology. 4. Results. 5. Discussion and conclusion. 6. Bibliography.

\section{Introducción. Hovland en la investigación en comunicación.}

Carl Iver Hovland, nació en Chicago el 12 de junio de 1912. Realizó sus estudios de grado en la Northwestern University y posteriormente se trasladó a la Universidad de Yale para realizar sus estudios de doctorado bajo la dirección de Clark L. Hull. Estudió los procesos de aprendizaje del ser humano y posteriormente se especializó en el área de psicología experimental. Su actividad investigadora en Yale fue interrumpida por la II Guerra Mundial.

En la Segunda Guerra Mundial, Estados Unidos se enfrenta a un serio problema de comunicación y persuasión. La guerra va a exigir que cientos de miles de jóvenes norteamericanos entren en combate en países lejanos. Las autoridades militares se ven obligadas a un esfuerzo de comunicación para explicar las causas de la guerra, para tratar de convencer al pueblo americano de la necesidad de que miles de jóvenes arriesguen su vida. Se hace un ingente esfuerzo dirigido a toda la población americana y a ello hay que sumar motivación y estímulo. Además era necesario mantener entre los soldados una moral alta.

Para este esfuerzo persuasivo el gobierno americano contrató a un equipo de científicos sociales y comunicadores para realizar esta tarea de persuasión sin precedentes.

En 1942, Hovland fue reclamado por el gobierno de Estados Unidos para coordinar los programas sobre el cambio de actitud en la Sección Experimental de la División de Información y Educación. Llevó a cabo diversos experimentos, sobre la eficacia y la influencia de determinados programas de capacitación y estudió los efectos persuasivos de una serie de documentales realizados por Frank Capra para los miembros del ejército. Una fuente privilegiada en este asunto es la autobiografía del propio autor, que bajo el título: "Frank Capra. El nombre delante del título", dedica dos capítulos a relatar todo lo acontecido en el periodo en que trabajó para el ejército de los Estados Unidos. De hecho, el capítulo XVII, tiene el mismo título que la serie de documentales que realizó "Why we figth" (Por qué combatimos). Capra describe cómo recibe el encargo del Jefe del Estado Mayor, el general George C. Marshall, en una reunión en la que le explica el objetivo para el que Capra era reclamado por el ejército: "Para ganar esta guerra debemos ganar la batalla de las mentes de los hombres. (...) Ahora, Capra deseo remachar con usted un plan para hacer una serie de filmes con información de facto, documentada, los primeros en nuestra historia, que explique a nuestros muchachos del Ejército por qué combatimos, y los principios por los que estamos luchando." (Capra, 2000: 337)

Una vez finalizada la guerra, Hovland, recopiló el material de investigación en el libro "Experiments on Mass Communication" (1949). Tras este periodo volvió a la Universidad de Yale donde trabajó al frente del Programa de Comunicación y Cambio de Actitud para 
investigar cómo cambian las opiniones de un destinatario en función de las variables manipuladas experimentalmente. A este periodo corresponden las obras "Communication and Persuassion" (1953), "The Order of Presentation in Persuasion" (1957) y "Personality and Persuasibility" (1959).

Hovland, no solo realizó investigaciones en el ámbito académico, sino que a finales de los 50 trabajó para los Bell Thelephone Laboratories. Formó parte de un grupo de investigación que realizaba investigaciones psicológicas en el ámbito industrial y trabajó junto con investigadores tan relevantes como Kurt Lewin.

Está considerado como uno de los cuatro padres del Mass Communication Research junto con Harold Laswell, Paul Lazarsfeld y Kurt Lewin. Schramm, en el prefacio de su obra "The Beginnings of Communication Study in America", explica que realiza esta obra con el fin de que los estudiantes conozcan la revolución que supuso para la investigación en comunicación la aparición de los estudios de Laswell, Lazarsfeld, Lewin y Hovland:

\begin{abstract}
"Estos «padres» de nuestro campo tuvieron carreras llamativamente semejantes. Tenían una espléndida formación, fueron a excelentes universidades, llegaron a contactar con grandes mentes. Todos eran ampliamente interdisciplinarios por inclinación. Sufrieron cambios de carrera abruptos en la mitad de su vida y derivaron de sus propias disciplinas a la comunicación por la experiencia de enfrentarse a problemas del «mundo real». Todos reunieron a su alrededor investigadores más jóvenes que llegaron a ser líderes del campo, y todos menos uno fundaron un instituto de investigación que atrajo a jóvenes brillantes y profesores capaces. Los cuatro fueron enormemente productivos". (Scramm, 1997: 25)
\end{abstract}

\title{
2. Experimentos aplicados a la comunicación.
}

Una de las más importantes aportaciones de Hovland a la investigación en comunicación fue el uso de experimentos. Como hemos señalado (Ruiz San Román, 2005) la cuestión del uso de experimentos en ciencias sociales no es pacífica y los autores han discutido largamente sobre la pertinencia y la posibilidad de hacer experimentos en ciencias sociales.

Sin embargo, aun teniendo en cuenta las dificultades de desarrollar diseños experimentales, Hovland mostró a la comunidad de investigadores en comunicación el enorme potencial de usar los experimentos en la investigación en comunicación.

Algunos de sus experimentos son incontestablemente parte de la historia de la investigación en comunicación y se han incorporado en los manuales de formación de generaciones enteras de graduados en comunicación. Por ejemplo, Klapper, en su esfuerzo por resumir lo más destacado de la investigación en comunicación hasta entonces, refiere los experimentos de Hovland con frecuencia (cfr. Klapper 1974: 89, 107, 110). Como es sabido, se trata de un texto muy utilizado en las universidades anglosajonas. Saperas (1985: 66 y ss), en su clásico texto "La sociología de la comunicación de masas en los Estados Unidos", explica con detalle los trabajos de Hovland y su equipo. O el más reciente texto de Lucas Marín (2000), en el campo de la Sociología de la Comunicacón, también se hace eco de las aportaciones de Hovland. En González (2010) mostramos la abundante presencia de las investigaciones de Hovland en los textos universitarios. En esa línea pueden estudiarse los textos de Mattelart (1997), Wolf (1987), etc. 


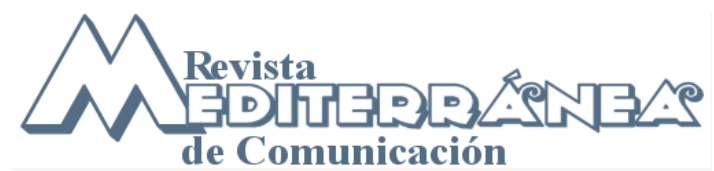

\section{Objeto y metodología.}

Nuestro objetivo es mostrar la influencia de Hovland en la investigación en comunicación en España. Nuestra hipótesis será que la importancia de las aportaciones de Hovland a la investigación debe tener su correlato en las citas de su obra en los trabajos posteriores. Nos limitaremos a la repercusión de la obra de Hovland en las revistas españolas y no serán objeto de nuestra investigación las referencias a su obra en otros países. Por tanto, será pertinente rastrear en la investigación en comunicación reciente si los trabajos del autor estudiado son citados por los investigadores actuales y en qué contexto se citan. De este modo se podría inferir la influencia actual de la obra de Hovland en nuestra comunidad científica de investigación en comunicación y, en cierto modo, si una de sus principales aportaciones, el uso de experimentos, está o no extendido entre nuestros investigadores. Para ello hemos hecho un estudio sistemático de la producción científica, un elemental estudio bibliométrico de las revistas de comunicación en nuestro país.

Nos pareció conveniente, siguiendo la metodología propuesta por Martínez Nicolás y Saperas (2011), hacer un vaciado de las publicaciones periódicas porque "constituyen el vehículo adecuado para la presentación de la investigación última, pues el lapso entre producción y publicación no suele demorarse en exceso. Son, por tanto, el indicador más fiable de las líneas de investigación abiertas, y allí se expresan de manera más actualizada los intereses de conocimiento de la comunidad científica en un momento determinado." (Martínez Nicolás y Saperas 2011: 106)

Centramos el análisis en la etapa más reciente porque nos interesa saber si la influencia de la obra de Hovland y sus propuestas metodológicas están influyendo y/o son conocidas por parte de los investigadores en comunicación de comienzos del siglo XXI.

Hicimos un vaciado de las principales revistas de comunicación con el objeto de detectar todas las citas o menciones a Hovland en el título, en el abstract o en el texto completo de los artículos.

Este artículo recoge los resultados de ese análisis de siete revistas españolas, que se corresponden con las revistas fuente de comunicación, tomando como referencia el índice de impacto de las revistas españolas de Ciencias Sociales en su apartado de Comunicación que elabora IN-RECS. Dichas revistas son: Anàlisi: quaderns de comunicació i cultura, Comunicación y Sociedad, Comunicar. Revista de Medios de Comunicación y Educación, Estudios sobre el Mensaje Periodístico, Revista Latina de Comunicación Social, Telos: Cuadernos de comunicación e innovación y Zer. Revista de Estudios de Comunicación.

En cuanto a la muestra utilizada se han tomado los contenidos de revistas fuente de comunicación en los años pares de la última década 2000, 2002, 2004, 2006, 2008 y 2010. Lo cual representa un $50 \%$ del universo de revistas científicas en comunicación consideradas revistas fuente en IN- RECS. 
Tabla 1: Corpus para el análisis de contenido.

\begin{tabular}{|c|c|c|c|c|c|c|c|c|c|c|c|c|c|c|c|}
\hline \multicolumn{16}{|c|}{ Corpus para el análisis de contenido } \\
\hline Año & \multicolumn{2}{|c|}{ Anàlisi } & \multicolumn{2}{|c|}{$\begin{array}{c}\text { Comunicación y } \\
\text { Sociedad }\end{array}$} & \multicolumn{2}{|c|}{ Comunicar } & \multicolumn{2}{|c|}{$\begin{array}{c}\text { Estudios sobre el } \\
\text { Mensaje } \\
\text { Periodístico }\end{array}$} & \multicolumn{2}{|c|}{$\begin{array}{c}\text { Revista Latina de } \\
\text { Comunicación } \\
\text { Social }\end{array}$} & \multicolumn{2}{|c|}{ Telos } & \multicolumn{2}{|c|}{ Zer } & $\begin{array}{l}\begin{array}{l}\text { Total } \\
\text { artículos } \\
\text { por año }\end{array} \\
\end{array}$ \\
\hline & $\begin{array}{c}\mathrm{N}^{\circ} \\
\text { Articulos } \\
\end{array}$ & Hovland & $\begin{array}{c}\mathrm{N}^{\circ} \\
\text { Articulos } \\
\end{array}$ & Hovland & $\begin{array}{c}\mathrm{N}^{\circ} \\
\text { Articulos } \\
\end{array}$ & Hovland & $\begin{array}{c}\mathrm{N}^{\circ} \\
\text { Articulos } \\
\end{array}$ & Hovland & $\begin{array}{c}\mathrm{N}^{\circ} \\
\text { Articulos } \\
\end{array}$ & Hovland & $\begin{array}{c}\mathrm{N}^{\circ} \\
\text { Articulos } \\
\end{array}$ & Hovland & $\begin{array}{c}\mathrm{N}^{\circ} \\
\text { Articulos } \\
\end{array}$ & Hovland & \\
\hline 2000 & 18 & 0 & 14 & 0 & 55 & 0 & 19 & 0 & 149 & 0 & * & & 29 & 0 & 284 \\
\hline 2002 & 25 & 1 & 11 & 0 & 60 & 0 & 23 & 0 & 88 & 0 & * & & 24 & 0 & 232 \\
\hline 2004 & 16 & 0 & 12 & 0 & 54 & 0 & 20 & 0 & 30 & 0 & 75 & 0 & 21 & 0 & 228 \\
\hline 2006 & 35 & 2 & 11 & 0 & 64 & 0 & 30 & 0 & 21 & 0 & 70 & 0 & 34 & 0 & 267 \\
\hline 2008 & 29 & 0 & 12 & 0 & 47 & 0 & 35 & 0 & 42 & 0 & 85 & 0 & 32 & 0 & 282 \\
\hline 2010 & 10 & 0 & 23 & 0 & 44 & 0 & 25 & 0 & 43 & 2 & 81 & 0 & 25 & 0 & 253 \\
\hline $\begin{array}{l}\text { total } \\
\text { artículos } \\
\text { por } \\
\text { revista }\end{array}$ & 133 & 3 & 83 & 0 & 324 & 0 & 152 & 0 & 373 & 2 & 311 & 0 & 165 & 0 & 1408 \\
\hline
\end{tabular}

\section{Resultados.}

En la muestra analizada nos encontramos con que de 1408 artículos, sólo 5 hacen referencia directa a Carl Hovland. Tres de estos artículos se encuentran en la revista Anàlisi, mientras que los dos restantes corresponden a la Revista Latina de Comunicación Social.

Los artículos son los siguientes:

- Anàlisi: quaderns de comunicació i cultura:

Gomis, L.; Martínez Albertos, J. L.; Núñez Ladéveze, L.; Casasús, J. M. (2002). "Encuesta: ¿vive la comunicación periodística un cambio de paradigma?". Anàlisi. Quaderns de comunicació i cultura, 28, p. 157-185.

Arceo, A. (2006). "Las relaciones públicas y la evaluación de la imagen proyectada por los medios de comunicación de masas Las elecciones a la presidencia de la Comunidad Autónoma de Madrid y al Ayuntamiento de Madrid en 2003". En Anàlisi. Quaderns de comunicació i cultura, 34, p. 239-254.

Martínez Nicolás, M. (2006). "Masa (en situación) crítica. La investigación sobre periodismo en España: comunidad científica e intereses de conocimiento". En Anàlisi. Quaderns de Comunicació i Cultura, 33, pp. 135-170.

\section{- Revista Latina de Comunicación Social:}

Piñuel, J. L. y Gaitán, J. A. (2010): "El discurso hegemónico sobre la verdad y la comunicación en la autorreferencia mediática en Prensa", en Revista Latina de Comunicación Social, 65. La Laguna (Tenerife): Universidad de La Laguna, páginas 572 a 594 recuperado el 3 de octubre de 2011, de http://www.revistalatinacs.org/10/art3/920_Complutense/42_Pinuel.html DOI: 10.4185/RLCS-66-2011-926-101-129 / CrossRef link 


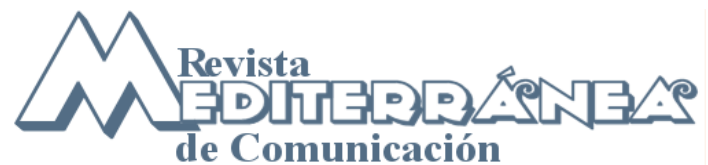

Idoyaga, Petxo et al (2010): Competencias interpretativas audiovisuales: entre la cultura textual y la alfabetización formalizada", en Revista Latina de Comunicación Social, 65. La Laguna (Tenerife): Universidad de La Laguna, páginas 266 a 277, recuperado el 3 de octubre de 2011, de http://www.revistalatinacs.org/10/art2/899_Bilbao/21_Idoyaga.html

En Gomis, Martínez Albertos, Núñez Ladeveze, Casasús (2002), Hovland es citado por el profesor Gomis como "pionero de la investigación en comunicación". Además expresamente se dice que es Schramm quien lo designa como uno de los primeros investigadores

En Arceo Vacas (2006), se cita a Hovland y se señala que el autor se considera "seguidor" de su obra (cfr. Arceo 2006: 217). De modo que toma de Hovland lo que considera de utilidad para su modelo de análisis. Martínez Nicolás (2006) cita a Hovland para señalar que "hasta comienzos de los ochenta la difusión en España de las aportaciones de las ciencias sociales al estudio de la comunicación y el periodismo se reducía prácticamente a los resultados de la mass communication research americana clásica - Lazarsfeld, Lasswell, Hovland, Berelson, Wright, Lewin-..." (M. Nicolás 2006: 151). En Piñuel y Gaitán (2010), citan a Hovland en su recorrido, para enmarcar históricamente la investigación en comunicación. Y finalmente, Idoyaga et al (2010) se refiere a Hovland como el autor que introduce el concepto clásico de "aprobación social".

Como se ve, son pocos los artículos que citan expresamente a Hovland. Sin embargo, el hecho de que medio siglo después de que la obra de Hovland se haya publicado se sigan encontrando citas expresas de su trabajo en las revistas especializadas, nos habla de su importancia e influencia. Consideramos que la mera presencia de un autor en las referencias de las revistas científicas cincuenta años después de su fallecimiento, es prueba suficiente de su notable repercusión.

A ello hay que añadir el dato que aportan Martínez Nicolás y Saperas sobre la escasa presencia de la metodología que Hovland promovió, el uso de experimentos en la investigación en comunicación. Sólo 3 artículos de los 179 estudiados, utilizan una técnica de investigación experimental, lo que supone el $3 \%$.

De alguna manera se puede decir que, aunque se conserva la memoria de la enorme importancia del autor, apenas se utilizan sus propuestas metodológicas de investigación mediante experimentos.

\section{Discusión y conclusión.}

Cincuenta años después de su muerte, Hovland pervive en la memoria de las diversas generaciones de investigadores en comunicación con una importante referencia histórica. Así, es considerado cita obligada para contextualizar explicaciones en la historia de la comunicación como hacen Piñuel y Gaitán (2010) o Martínez Nicolás y Saperas (2006). Sin embargo, lo que consideramos su principal aportación metodológica, no se cita en las revistas de investigación. Nadie refiere los experimentos de Yale como antecedentes de su investigación.

A nuestro juicio, esto pone de manifiesto una debilidad de la actual investigación en comunicación: la falta de seguimiento de una de las tradiciones investigadoras más rigurosas en ciencias sociales, los experimentos. Muy utilizados, por ejemplo, en la psicología social. 


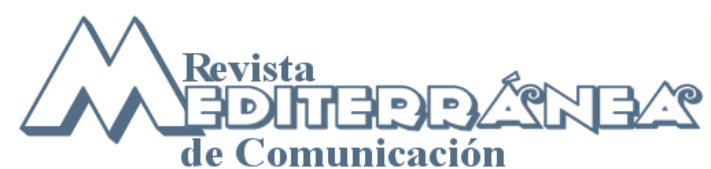

ISSN 1989-872X - Año3 (2012), pp. 31-38

Podría argumentarse que se está utilizando la investigación con experimentos, pero que no se cita necesariamente a Hovland. Sin embargo, las referidas investigaciones recientes de Martínez Nicolás y Saperas (2011), descartan esta hipótesis; porque dejan claro que los experimentos apenas se usan.

Como hemos señalado en otras ocasiones, es cierto que los experimentos pueden ser contemplados como "una técnica complicada; pero de darse, muy útil, pues aporta luces sobre las relaciones causales" (Ruíz San Román, 2005: 236).

Por tanto, subrayamos la necesidad de que se fomente la investigación mediante el uso de experimentos en comunicación en la línea iniciada hace más de medio siglo por Carl I. Hovland.

\section{Bibliografía.}

Arceo, A. (2006). "Las relaciones públicas y la evaluación de la imagen proyectada por los medios de comunicación de masas Las elecciones a la presidencia de la Comunidad Autónoma de Madrid y al Ayuntamiento de Madrid en 2003". En Anàlisi. Quaderns de comunicació i cultura, 34, p. 239-254

Berganza Conde, M. R y Ruiz San Román J. A (Coord). (2005). Investigar en comunicación: guía práctica de métodos y técnicas de investigación social en comunicación. Madrid: McGraw Hill.

Capra, F. (2000). Frank Capra. El nombre delante del título. Autobiografía. Madrid: T\&B

Gomis, L.; Martínez Albertos, J. L.; Núñez Ladéveze, L.; Casasús, J. M. (2002). “Encuesta: ¿vive la comunicación periodística un cambio de paradigma?". Anàlisi. Quaderns de comunicació i cultura, 28, p. 157-185

Idoyaga, Petxo et al (2010): Competencias interpretativas audiovisuales: entre la cultura textual y la alfabetización formalizada", en Revista Latina de Comunicación Social, 65. La Laguna (Tenerife): Universidad de La Laguna, páginas 266 a 277, recuperado el 3 de octubre de 2011, de http://www.revistalatinacs.org/10/art2/899_Bilbao/21_Idoyaga.html DOI: 10.4185/RLCS-65-2010-899-266-277

Hovland, C; Lumsdaine, A y Sheffielf, F. (1965). Experiments on Mass Communication. NewYork: Science Editions. (Versión original 1949).

Hovland, C; Janis, I y Kelley, H. (1966). Communication and Persuasion. New Haven: Yale University Press. (Versión original 1953).

Hovland, C; MAndell, W; Campbell, E.H; Brock, T; Luchins, A.S; Cohen, A.R; McGuire, W.J; Janis, I.L; Feierabend, R.L y Anderson, N.H (1957) The order of presentation in persuasion. New Haven: Yale University Press.

Hovland, C; Janis, I; Field, P; Linton, H; Graham, E; Cohen, A; Rife, D; Abelson, R; Leser, G y King, B (1959) Personality and Persuasibility. New Haven: Yale University Press.

Klapper, J.(1974). Efectos de las comunicaciones de masas: poder y limitaciones de los medios modernos de difusión. Madrid: Aguilar. 
Lucas Marín, A (ed); García Galera, C, Linares Rodríguez, V; Martín Cárdaba, M. A; Ruiz San Román J. A y Vinuesa Tejero, M. L (2009) La Nueva Comunicación. Madrid: Trotta.

Martínez Nicolás, M. (2006). "Masa (en situación) crítica. La investigación sobre periodismo en España: comunidad científica e intereses de conocimiento". En Anàlisi. Quaderns de Comunicació i Cultura, 33, pp. 135-170.

Martínez Nicolás, M. y Saperas Lapiedra, E. (2011): "La investigación sobre Comunicación en España (1998-2007). Análisis de los artículos publicados en revistas científicas", en Revista Latina de Comunicación Social, 66. La Laguna (Tenerife): Universidad de La Laguna, páginas 101 a 129 recuperado el 4 de febrero de 2011, de http://www.revistalatinacs.org/11/art/926_Vicalvaro/05_Nicolas.html DOI: 10.4185/RLCS-66-2011-926-101-129 / CrossRef link

Mattelart, A y Mattelart, M. (1997) Historia de las teorías de la comunicación. Barcelona: Paidós.

Piñuel, J. L. y Gaitán, J. A. (2010): "El discurso hegemónico sobre la verdad y la comunicación en la autorreferencia mediática en Prensa", en Revista Latina de Comunicación Social, 65. La Laguna (Tenerife): Universidad de La Laguna, páginas 572 a 594 recuperado el 3 de octubre de 2011, de http://www.revistalatinacs.org/10/art3/920_Complutense/42_Pinuel.html

DOI: 10.4185/RLCS-66-2011-926-101-129 / CrossRef link

Saperas, E. (1985). La sociología de la comunicación de masas en los Estados Unidos. Barcelona: Ariel.

Shepard, R. (1998). Carl Iver Hovland. En Biographical Memoirs. Vol. 73 (pp. 230-261). Washington D.C: National Academy Press.

Schramm, W. (1997). The Beginnings of Communication Study in America: a Personal Memoir. Thousand Oaks, California: Sage Publications.

Wolf, M. (1987). La investigación de la comunicación de masas. Crítica y perspectivas. Barcelona: Paidós. 